\title{
Hermite interpolating polynomials
}

\author{
A. Nyíri
}




\title{
HERMITE INTERPOLATING POLYNOMIALS
}

\author{
A. NYÍRI
}

[Received: January 30, 2004]

Abstract. A method is described to construct Hermite polynomials fitting on to 2, 4,8 points of a 1D, 2D, 3D functions, respectively, up to their $r$ th derivatives.

Mathematics Subject Classification: 65D05

Keywords: 1D, 2D, 3D Hermite polynomials

\section{InTRODUCTION}

The Hermite interpolation is a well-known theoretical tool for approximating functions. Although the theory and practice of Hermite interpolation is well developed for univariate functions (see, e. g., $[1,4,6]$ ), fewer results is known for the multivariate case (see, e. g., [3,5]). We emphasize the importance of the work of Lorentz [3], which surveys the well-known theory on the multivariate case. Here we describe a computational method to construct Hermite polynomials fitting on to 2, 4, 8 points of a 1D, 2D, 3D functions, respectively, up to their $r$ th derivatives.

\section{ONE-DiMENSIONAL POLYNOMIAL}

The aim of this section is to construct a polynomial $H_{r}(x)$ on to the points $x_{0}$ and $x_{1}$ of the function $y(x)$ up to its $r$ th derivatives.

$$
\frac{d^{m} H_{r}\left(x_{l}\right)}{d x^{m}}=\frac{d^{m} y\left(x_{l}\right)}{d x^{m}}, \quad l=0,1, \quad 0 \leq m \leq r .
$$

Let us seek for it in the form

$$
H_{r}(x)=\boldsymbol{A}_{r, 1} \boldsymbol{X}_{r, 1}(x)+\boldsymbol{A}_{r, 2} C_{r}(x) \boldsymbol{X}_{r, 2}(x) .
$$

The number of the prescribed conditions is $2 r+2$. For the sake of a convenient form of writing the vectors, we define the $\boldsymbol{e}_{r, j+1}$ unit vector $(0 \leq j \leq r)$ the $(j+1)$ th entry of which is equal to 1 and the rest are equal zero. Then the vectors in (1) can 
be written in the form

$$
\begin{aligned}
\boldsymbol{A}_{r, 1} & =\sum_{j=0}^{r} a_{j+1} \boldsymbol{e}_{r, j+1}, & \boldsymbol{A}_{r, 2} & =\sum_{j=0}^{r} a_{r+2+j} \boldsymbol{e}_{r, j+1}, \\
\boldsymbol{X}_{r, 1}(x) & =\sum_{j=0}^{r}\left(x-x_{0}\right)^{j} \boldsymbol{e}_{r, j+1}, & \boldsymbol{X}_{r, 2}(x) & =\sum_{j=0}^{r}\left(x-x_{1}\right)^{j} \boldsymbol{e}_{r, j+1},
\end{aligned}
$$

where $C_{r}(x)=\left(x-x_{0}\right)^{r+1}$. To determine the constants $a$, the derivatives of the function $H_{r}(x)$ must be calculated at the values $x_{0}$ and $x_{1}$,

$$
\begin{aligned}
\boldsymbol{A}_{r, 1} \boldsymbol{X}_{r, 1}(x) & =\sum_{j=0}^{r} a_{j+1}\left(x-x_{0}\right)^{j}, \\
\boldsymbol{A}_{r, 1} \frac{d^{m} \boldsymbol{X}_{r, 1}(x)}{d x^{m}} & =\sum_{j=0}^{r-m} \frac{(m+j) !}{j !}\left(x-x_{0}\right)^{j} a_{m+1+j},
\end{aligned}
$$

Let us set $x_{1}-x_{0}=h$. Then

$$
\begin{aligned}
& \boldsymbol{A}_{r, 1} \frac{d^{m} \boldsymbol{X}_{r, 1}\left(x_{0}\right)}{d x^{m}}=m ! a_{m+1}, \\
& \boldsymbol{A}_{r, 1} \frac{d^{m} \boldsymbol{X}_{r, 1}\left(x_{1}\right)}{d x^{m}}=\sum_{j=0}^{r-m} \frac{(m+j) !}{j !} h^{j} a_{m+1+j}, \quad 0 \leq m \leq r .
\end{aligned}
$$

To compute the derivatives of the second term in $H_{r}(x)$, the following notation will be used: $f_{r}(x)=\left(x-x_{0}\right)^{r+1}, g_{j}(x)=\left(x-x_{1}\right)^{j}$,

$$
\frac{d^{t} f_{r}(x)}{d x^{t}}=f_{r}^{(t)}(x)=\frac{(r+1) !}{(r+1-t) !}\left(x-x_{0}\right)^{r+1-t},
$$

$f_{r}^{(r+1)}(x)=(r+1) !, f_{r}^{(t)}\left(x_{0}\right)=0$ if $t<r+1$. Let $t=m-l$. Then $f_{r}^{(m-l)}\left(x_{0}\right)=0$ if $r+1>m-l$, and

moreover,

$$
f_{r}^{(m-l)}\left(x_{1}\right)=\frac{(r+1) !}{(r+1-m+l) !} h^{r+1-m+l}, \quad m-l \leq r+1 ;
$$

$$
\begin{aligned}
g_{j}^{(l)}(x) & =\frac{j !}{(j-l) !}\left(x-x_{1}\right)^{j-l}, \quad l \leq j, \quad g_{j}^{(j)}(x)=j !, \\
g_{j}^{(l)}\left(x_{1}\right) & = \begin{cases}0 & \text { if } l \neq j, \\
j ! & \text { if } l=j .\end{cases}
\end{aligned}
$$

The derivative of their product is

$$
\frac{d^{m}}{d x^{m}}\left[f_{r}(x) g_{j}(x)\right]=\sum_{l=0}^{m}\left(\begin{array}{c}
m \\
l
\end{array}\right) f_{r}^{(m-l)}(x) g_{j}^{(l)}(x),
$$


and $\left(d^{m} / d x^{m}\right)\left[f_{r}\left(x_{0}\right) g_{j}\left(x_{0}\right)\right]=0$ for $0 \leq m \leq r$.

One has $g_{j}^{(l)}\left(x_{1}\right)=0$ if $l \neq j$ and $g_{j}^{(j)}=j !$; therefore $m-l=m-j$, and then

$$
f_{r}^{(m-j)}\left(x_{1}\right)=\frac{(r+1) !}{(r+1-m+j) !} h^{r+1-m+j}, \quad 0 \leq m \leq r .
$$

The derivative at the point $x_{1}$ will be

$$
\frac{d^{m}}{d x^{m}}\left[f_{r}\left(x_{1}\right) g_{j}\left(x_{1}\right)\right]=\sum_{j=0}^{m} \frac{m !}{(m-j) !} \frac{(r+1) !}{(r+1-m+j) !} h^{r+1-m+j}, \quad 0 \leq m \leq r,
$$

and at $x_{0}$

$$
\frac{d^{m}}{d x^{m}}\left[f_{r}\left(x_{0}\right) g_{j}\left(x_{0}\right)\right]=\sum_{l=l_{\min }}^{l_{\max }}\left(\begin{array}{c}
m \\
l
\end{array}\right) f_{r}^{(m-l)}\left(x_{0}\right) g_{j}^{(l)}\left(x_{0}\right) .
$$

If $m-l \neq r+1$, then $f_{r}^{(m-l)}\left(x_{0}\right)=0$ and $f_{r}^{(r+1)}\left(x_{0}\right)=(r+1)$ !, so $m-l=r+1$, $l=m-(r+1)$, and

$$
g_{j}^{(m-(r+1))}\left(x_{0}\right)=\frac{j !}{(r+1-m+j) !}(-h)^{r+1-m+j},
$$

whence

$$
\begin{aligned}
\frac{d^{m}}{d x^{m}}\left[f_{r}\left(x_{0}\right) g_{j}\left(x_{0}\right)\right] & = \\
& =\left(\begin{array}{c}
m \\
m-(r+1)
\end{array}\right)(r+1) ! \sum_{j=m-(r+1)}^{r} \frac{j !}{(r+1-m+j) !}(-h)^{r+1-m+j}
\end{aligned}
$$

for $r+1 \leq m \leq 2 r+1$. The derivatives at the point $x_{1}$ are $g_{j}^{(l)}\left(x_{1}\right)=0$ if $l \neq j$, therefore, with $m-l=m-j$, there should be

$$
f_{r}^{(m-j)}\left(x_{1}\right)=\frac{(r+1) !}{(r+1-m+j) !} h^{r+1-m+j},
$$

whence

$$
\frac{d^{m}}{d x^{m}}\left[f_{r}\left(x_{1}\right) g_{j}\left(x_{1}\right)\right]==m !(r+1) ! \sum_{j=m-(r+1)}^{r} \frac{h^{r+1-m+j}}{(m-j) !(r+1-m+j) !}
$$

for $r+1 \leq m \leq 2 r+1$. Returning to the original notation, we get

$$
C_{r}(x) \boldsymbol{A}_{r, 2} \boldsymbol{X}_{r, 2}(x)=\sum_{j=0}^{r} a_{r+2+j} f_{r}(x) g_{j}(x) .
$$


Summing up the results above, we get

$$
\begin{aligned}
& \boldsymbol{A}_{r, 1} \frac{d^{m} \boldsymbol{X}_{r, 1}\left(x_{0}\right)}{d x^{m}}=m ! a_{m+1}, \quad 0 \leq m \leq r, \\
& \boldsymbol{A}_{r, 1} \frac{d^{m} \boldsymbol{X}_{r, 1}\left(x_{1}\right)}{d x^{m}}=\sum_{j=0}^{r-m} \frac{(m+j) !}{j !} h^{j} a_{m+1+j}, \quad 0 \leq m \leq r, \\
& \boldsymbol{A}_{r, 1} \frac{d^{m} \boldsymbol{X}_{r, 1}\left(x_{0}\right)}{d x^{m}}=0, \quad m>r, \\
& \boldsymbol{A}_{r, 2} \frac{d^{m}}{d x^{m}}\left[C_{r}\left(x_{0}\right) \boldsymbol{X}_{r, 2}\left(x_{0}\right)\right]=0, \quad 0 \leq m \leq r, \\
& \boldsymbol{A}_{r, 2} \frac{d^{m}}{d x^{m}}\left[C_{r}\left(x_{1}\right) \boldsymbol{X}_{r, 2}\left(x_{1}\right)\right]=m !(r+1) ! \sum_{j=0}^{m} \frac{h^{r+1-m+j}}{(m-j) !(r+1-m+j) !} a_{r+2+j} \\
& \text { for } 0 \leq m \leq r \text {, } \\
& \boldsymbol{A}_{r, 2} \frac{d^{m}}{d x^{m}}\left[C_{r}\left(x_{0}\right) \boldsymbol{X}_{r, 2}\left(x_{0}\right)\right]=\left(\begin{array}{c}
m \\
m-(r+1)
\end{array}\right)(r+1) ! \times \\
& \times \sum_{j=m-(r+1)}^{r} \frac{j !(-h)^{r+1-m+j}}{(r+1-m+j) !} a_{r+2+j} \\
& \boldsymbol{A}_{r, 2} \frac{d^{m}}{d x^{m}}\left[C_{r}\left(x_{1}\right) \boldsymbol{X}_{r, 2}\left(x_{1}\right)\right]=m !(r+1) ! \sum_{j=m-(r+1)}^{r} \frac{h^{r+1-m+j}}{(m-j) !(r+1-m+j) !} a_{r+2+j} \\
& \frac{d^{m} H_{r}(x)}{d x^{m}}=\boldsymbol{A}_{r, 1} \frac{d^{m} \boldsymbol{X}_{r, 1}}{d x^{m}}+\boldsymbol{A}_{r, 2} \frac{d^{m}}{d x^{m}}\left[C_{r}(x) \boldsymbol{X}_{r, 2}(x)\right] .
\end{aligned}
$$

Example 1. For $r=2$,

$$
\begin{aligned}
H_{2}\left(x_{0}\right) & =a_{1}, \\
\frac{d H_{2}\left(x_{0}\right)}{d x} & =a_{2}, \\
\frac{d^{2} H_{2}\left(x_{0}\right)}{d x^{2}} & =a_{3} 2, \\
H_{2}\left(x_{1}\right) & =a_{1}+a_{2} h+a_{3} h^{2}+a_{4} h^{3}, \\
\frac{d H_{2}\left(x_{1}\right)}{d x} & =a_{2}+a_{3} 2 h+a_{4} 3 h^{2}+a_{5} h^{3}, \\
\frac{d^{2} H_{2}\left(x_{1}\right)}{d x^{2}} & =a_{3} 2+a_{4} 6 h+a_{5} 6 h^{2}+a_{6} 2 h^{3} .
\end{aligned}
$$




\section{Two-dimensional Polynomial}

The aim of this section is to construct a polynomial $H_{r}(x, y)$ on to the points $\left(x_{0}, y_{0}\right),\left(x_{0}, y_{1}\right),\left(x_{1}, y_{0}\right),\left(x_{1}, y_{1}\right)$, which fit the given values up to its $r$ th derivatives. The conditions can be written as follows:

$$
\frac{\partial^{m+n} H_{r}\left(x_{s}, y_{t}\right)}{\partial x^{m} \partial y^{n}}=\frac{\partial^{m+n} u\left(x_{s}, y_{t}\right)}{\partial x^{m} \partial y^{n}}
$$

for $0 \leq m, n \leq r, 0 \leq m+n \leq r, s, t=0,1$. The number of the prescribed conditions at one point is $\frac{1}{2} r(r+3)+1$, and in the four points is $2 r^{2}+6 r+4$.

Let us seek for it in the form

$$
H_{r}(x, y)=\boldsymbol{A}(x) \boldsymbol{Y}_{r, 1}(y)+\boldsymbol{B}(x) D_{r}(y) \boldsymbol{Y}_{r, 2}(y) .
$$

For the sake of a convenient form of writing the vectors, let us define the unit vector of $j+1$ entries, $\boldsymbol{e}_{j, q+1}(0 \leq q \leq j)$, the $(q+1)$ th entry of which is equal to 1 and the rest are equal to zero. Then the vectors in $H_{r}(x, y)$ can be written as follows:

$$
\begin{aligned}
\boldsymbol{A}(x) & =\sum_{j=0}^{r} A_{j}(x) \boldsymbol{e}_{r, r+1-j}, & \boldsymbol{B}(x) & =\sum_{j=0}^{r} B_{j}(x) \boldsymbol{e}_{r, r+1-j}, \\
\boldsymbol{Y}_{j, 1}(y) & =\sum_{i=0}^{j}\left(y-y_{0}\right)^{i} \boldsymbol{e}_{j, i+1}, & \boldsymbol{Y}_{j, 2}(y) & =\sum_{i=0}^{j}\left(y-y_{1}\right)^{i} \boldsymbol{e}_{j, i+1},
\end{aligned}
$$

where $D_{j}(y)=\left(y-y_{0}\right)^{j+1}, 0 \leq j \leq r$. The functions $\boldsymbol{A}(x)$ and $\boldsymbol{B}(x)$ are composed as

$$
\begin{aligned}
A_{j}(x) & =\boldsymbol{A}_{j, 1} \boldsymbol{X}_{j, 1}(x)+\boldsymbol{A}_{j, 2} C_{j}(x) \boldsymbol{X}_{j, 2}(x), \\
B_{j}(x) & =\boldsymbol{B}_{j, 1} \boldsymbol{X}_{j, 1}(x)+\boldsymbol{B}_{j, 2} C_{j}(x) \boldsymbol{X}_{j, 2}(x),
\end{aligned}
$$

where

$$
\boldsymbol{X}_{j, 1}(x)=\sum_{i=0}^{j}\left(x-x_{0}\right)^{i} \boldsymbol{e}_{j, i+1}, \quad \boldsymbol{X}_{j, 2}(x)=\sum_{i=0}^{j}\left(x-x_{1}\right)^{i} \boldsymbol{e}_{j, i+1},
$$

and $C_{j}(x)=\left(x-x_{0}\right)^{j+1}, 0 \leq j \leq r$. The distances between the coordinates are $x_{1}-x_{0}=h, y_{1}-y_{0}=g$.

For writing the vectors $\boldsymbol{A}_{j, 1}, \boldsymbol{A}_{j, 2}, \boldsymbol{B}_{j, 1}, \boldsymbol{B}_{j, 2}$ conveniently, we introduce the notation

$$
l_{j}=(r-j) \frac{r+3+j}{2}, \quad n_{r}=1 / 2(r+1)(r+2), \quad L_{j}=l_{j}+n_{r} .
$$

With this notation, we get

$$
\begin{aligned}
\boldsymbol{A}_{j, 1} & =\sum_{i=0}^{j} a_{l_{j}+i+1} \boldsymbol{e}_{j, i+1}, & \boldsymbol{A}_{j, 2} & =\sum_{i=0}^{j} a_{L_{j}+i+1} \boldsymbol{e}_{j, i+1}, \\
\boldsymbol{B}_{j, 1} & =\sum_{i=0}^{j} b_{l_{j}+i+1} \boldsymbol{e}_{j, i+1}, & \boldsymbol{B}_{j, 2} & =\sum_{i=0}^{j} b_{L_{j}+i+1} \boldsymbol{e}_{j, i+1} .
\end{aligned}
$$


The number of the constants $a, b$ in $\boldsymbol{A}(x), \boldsymbol{B}(x)$ is $4 n_{r}=2 r^{2}+6 r+4$, which agrees with the number of conditions; consequently, $H_{r}(x, y)$ is the minimal degree polynomial satisfying the requirements.

The derivatives of the above vectors should be computed,

$$
\begin{aligned}
\frac{d^{m} \boldsymbol{X}_{j, 1}(x)}{d x^{m}} & =\sum_{q=0}^{j-m} \frac{(m+q) !}{q !}\left(x-x_{0}\right)^{q} \boldsymbol{e}_{j, m+1+q}, & & 0 \leq m \leq j, \\
\frac{d^{t} \boldsymbol{X}_{j, 2}(x)}{d x^{t}} & =\sum_{q=0}^{j-t} \frac{(t+q) !}{q !}\left(x-x_{1}\right)^{q} \boldsymbol{e}_{j, t+1+q}, & & 0 \leq t \leq j, \\
\frac{d^{l} C_{j}(x)}{d x^{l}} & =C_{j}^{(l)}(x)=\frac{(j+1) !}{(j+1-l) !}\left(x-x_{0}\right)^{j+1-l}, & & 0 \leq l \leq j+1, \\
\frac{d^{m}}{d x^{m}}\left[C_{j}(x) \boldsymbol{X}_{j, 2}(x)\right] & =\sum_{l=l_{\min }}^{l_{\max }}\left(\begin{array}{c}
m \\
l
\end{array}\right) C_{j}^{(l)}(x) \boldsymbol{X}_{j, 2}^{(m-l)}(x) . & &
\end{aligned}
$$

The limits of the sums are

$$
\begin{array}{ll}
l_{\min }=0, l_{\max }=m & \text { if } 0 \leq m \leq j, \\
l_{\min }=m-j, l_{\max }=j+1 & \text { if } j+1 \leq m \leq 2 j+1 .
\end{array}
$$

The derivatives of $A_{j}(x)$ and $B_{j}(x)$ are

$$
\begin{aligned}
\frac{d^{m} A_{j}(x)}{d x^{m}} & =\boldsymbol{A}_{j, 1} \frac{d^{m} \boldsymbol{X}_{j, 1}(x)}{d x^{m}}+\boldsymbol{A}_{j, 2} \frac{d^{m}}{d x^{m}}\left[C_{j}(x) \boldsymbol{X}_{j, 2}(x)\right], \\
\frac{d^{m} B_{j}(x)}{d x^{m}} & =\boldsymbol{B}_{j, 1} \frac{d^{m} \boldsymbol{X}_{j, 1}(x)}{d x^{m}}+\boldsymbol{B}_{j, 2} \frac{d^{m}}{d x^{m}}\left[C_{j}(x) \boldsymbol{X}_{j, 2}(x)\right] .
\end{aligned}
$$

To compute the coefficients $a$ and $b$ at the points $x_{0}$ and $x_{1}$, the following formulas are used:

$$
\begin{array}{rlr}
\frac{d^{m} A_{j}\left(x_{0}\right)}{d x^{m}} & =m ! a_{l_{j}+m+1}, & 0 \leq m \leq j, \\
\frac{d^{m} A_{j}\left(x_{1}\right)}{d x^{m}}= & \sum_{q=0}^{j-m} \frac{(m+q) !}{q !} h^{q} a_{l_{j}+m+1+q}+ & \\
& +m ! \sum_{l=0}^{m} \frac{(j+1) !}{l !(j+1-l) !} h^{j+1-l} a_{L_{j}+m+1-l}, & 0 \leq m \leq j, \\
\frac{d^{m} A_{j}\left(x_{0}\right)}{d x^{m}}= & \frac{m !}{[m-(j+1)] !} \times & \\
& \times \sum_{q=0}^{2 j+1-m} \frac{[m-(j+1)+q] !}{q !}(-h)^{q} a_{L_{j}+m-j+q},
\end{array}
$$




$$
j+1 \leq m \leq 2 j+1,
$$

$$
\begin{gathered}
\frac{d^{m} A_{j}\left(x_{1}\right)}{d x^{m}}=m ! \sum_{l=m-j}^{j+1} \frac{(j+1) !}{(j+1-l) !} h^{j+1-l} a_{L_{j}+m+1-l} \\
j+1 \leq m \leq 2 j+1 .
\end{gathered}
$$

Analogous formulas are valid for $B_{j}(x)$. The derivatives of the Hermite polynomial are

$$
\frac{\partial^{m+n} H_{r}(x, y)}{\partial x^{m} \partial y^{n}}=\frac{d^{m} \boldsymbol{A}(x)}{d x^{m}} \frac{d^{n} \boldsymbol{Y}_{r, 1}(y)}{d y^{n}}+\frac{d^{m} \boldsymbol{B}(x)}{d x^{m}} \frac{d^{n}}{d y^{n}}\left[D_{r}(y) \boldsymbol{Y}_{r, 2}(y)\right] .
$$

The following formulas are also needed:

$$
\begin{aligned}
\frac{d^{n} \boldsymbol{Y}_{j, 1}(y)}{d y^{n}} & =\sum_{s=0}^{j-n} \frac{(n+s) !}{s !}\left(y-y_{0}\right)^{s} \boldsymbol{e}_{j, n+1+s}, & & 0 \leq n \leq j, \\
\frac{d^{t} \boldsymbol{Y}_{j, 2}(y)}{d y^{t}} & =\sum_{s=0}^{j-t} \frac{(t+s) !}{s !}\left(y-y_{1}\right)^{s} \boldsymbol{e}_{j, t+1+s}, & & 0 \leq t \leq j, \\
\frac{d^{l} D_{j}(y)}{d y^{l}} & =D_{j}^{(l)}(y)=\frac{(j+1) !}{(j+1-l) !}\left(y-y_{0}\right)^{j+1-l}, & & 0 \leq l \leq j+1 .
\end{aligned}
$$

Thus, we can write

$$
\frac{\partial^{m+n}}{\partial x^{m} \partial y^{n}}\left[\boldsymbol{A}(x) \boldsymbol{Y}_{r, 1}(y)\right]=\sum_{j=0}^{r-n} A_{j}(x) \frac{(r-j) !}{(r-n-j) !}\left(y-y_{0}\right)^{r-n-j},
$$

and the values of these are

$$
\begin{aligned}
\frac{\partial^{m+n}}{\partial x^{m} \partial y^{n}}\left[\boldsymbol{A}\left(x_{0}\right) \boldsymbol{Y}_{r, 1}\left(y_{0}\right)\right] & =n ! \frac{d^{m} A_{r-n}\left(x_{0}\right)}{d x^{m}}, \\
\frac{\partial^{m+n}}{\partial x^{m} \partial y^{n}}\left[\boldsymbol{A}\left(x_{0}\right) \boldsymbol{Y}_{r, 1}\left(y_{1}\right)\right] & =\sum_{j=0}^{r-n} \frac{d^{m} A_{j}\left(x_{0}\right)}{d x^{m}} \frac{(r-j) !}{(r-n-j) !} g^{r-n-j}, \\
\frac{\partial^{m+n}}{\partial x^{m} \partial y^{n}}\left[\boldsymbol{A}\left(x_{1}\right) \boldsymbol{Y}_{r, 1}\left(y_{0}\right)\right] & =n ! \frac{d^{m} A_{r-n}\left(x_{1}\right)}{d x^{m}}, \\
\frac{\partial^{m+n}}{\partial x^{m} \partial y^{n}}\left[\boldsymbol{A}\left(x_{1}\right) \boldsymbol{Y}_{r, 1}\left(y_{1}\right)\right] & =\sum_{j=0}^{r-n} \frac{d^{m} A_{j}\left(x_{1}\right)}{d x^{m}} \frac{(r-j) !}{(r-n-j) !} g^{r-n-j}
\end{aligned}
$$

for $0 \leq n \leq r, 0 \leq m \leq 2 r+1$.

To compute the derivatives of the second term in $H_{r}(x, z)$, the next interim notation will be used: $f_{r}(y)=D_{r}(y), g_{j}(y)=\left(y-y_{1}\right)^{r-j}$. The derivatives of these are

$$
f_{r}^{(l)}(y)=\frac{(r+1) !}{(r+1-l) !}\left(y-y_{0}\right)^{r+1-l}, \quad 0 \leq l \leq r+1,
$$


$f_{r}^{(r+1)}(y)=(r+1) !, f_{r}^{(l)}\left(y_{0}\right)=0$ if $0 \leq l<r+1, f_{r}^{(l)}(y)=0$ if $l>r+1, g_{j}^{(t)}(y)=$ $(r-j) !((r-j-t) !)^{-1}\left(y-y_{1}\right)^{r-j-t}$,

$$
\begin{aligned}
g_{j}^{(t)}(y) & = \begin{cases}(r-j) ! & \text { if } t=r-j, \\
0 & \text { if } t \neq r-j,\end{cases} \\
\frac{d^{n} f_{r}(y)}{d y^{n}} & =\sum_{l=l_{\min }}^{l_{\max }}\left(\begin{array}{l}
n \\
l
\end{array}\right) f_{r}^{(l)}(y) g_{j}^{(n-l)}(y),
\end{aligned}
$$

$l_{\min }=\max \{n-(r-j), 0\}, l_{\max }=n$ if $r-n \leq j \leq r$ and $0 \leq n \leq r ; l_{\min }=n+j-r$, $l_{\max }=r+1$ if $0 \leq j \leq 2 r+1-n, r+1 \leq n \leq 2 r+1$. It is easy to see that

$$
\begin{array}{ll}
\frac{d^{n}}{d y^{n}}\left[f_{r}\left(y_{0}\right) g_{j}\left(y_{0}\right)\right]=0, & 0 \leq n \leq r, \\
\frac{d^{n}}{d y^{n}}\left[f_{r}\left(y_{1}\right) g_{j}\left(y_{1}\right)\right]=\left(\begin{array}{c}
n \\
n+j-r
\end{array}\right) \frac{(r+1) !(r-j) !}{(2 r+1-n-j) !} g^{2 r+1-n-j}, & 0 \leq n \leq r, r-n \leq j \leq r,
\end{array}
$$

$$
\frac{d^{n}}{d y^{n}}\left[f_{r}\left(y_{0}\right) g_{j}\left(y_{0}\right)\right]=\left(\begin{array}{c}
n \\
r+1
\end{array}\right) \frac{(r+1) !(r-j) !}{(2 r+1-n-j) !}(-g)^{2 r+1-n-j}
$$

for $r+1 \leq n \leq 2 r+1,0 \leq j \leq 2 r+1-n$,

$$
\frac{d^{n}}{d y^{n}}\left[f_{r}\left(y_{1}\right) g_{j}\left(y_{1}\right)\right]=\left(\begin{array}{c}
n \\
n+j-r
\end{array}\right) \frac{(r+1) !(r-j) !}{(2 r+1-n-j) !} g^{2 r+1-n-j}
$$

for $r+1 \leq n \leq 2 r+1,0 \leq j \leq 2 r+1-n$. The derivatives of the second term in $H_{r}(x, y)$ are

$$
\frac{\partial^{m+n}}{\partial x^{m} \partial y^{n}}\left[\boldsymbol{B}(x) D_{r}(y) \boldsymbol{Y}_{r, 2}(y)\right]=\sum_{j=0}^{r} \frac{d^{m} B_{j}(x)}{d x^{m}} \frac{d^{n}}{d y^{n}}\left[f_{r}(y) g_{j}(y)\right],
$$

and their values of at $x, y_{0}$ and $y_{1}$ are

$$
\frac{\partial^{m+n}}{\partial x^{m} \partial y^{n}}\left[\boldsymbol{B}(x) D_{r}\left(y_{0}\right) \boldsymbol{Y}_{r, 2}\left(y_{0}\right)\right]=0
$$

for $0 \leq m \leq 2 r+1,0 \leq n \leq r$;

$$
\begin{aligned}
\frac{\partial^{m+n}}{\partial x^{m} \partial y^{n}}\left[\boldsymbol{B}(x) D_{r}\left(y_{1}\right) \boldsymbol{Y}_{r, 2}\left(y_{1}\right)\right] & =(r+1) ! \sum_{j=r-n}^{r} \frac{d^{m} B_{j}(x)}{d x^{m}} \times \\
& \times\left(\begin{array}{c}
n \\
n+j-r
\end{array}\right) \frac{(r-j) !}{(2 r+1-n-j) !} g^{2 r+1-n-j}
\end{aligned}
$$


for $0 \leq m \leq 2 r+1,0 \leq n \leq r$

$$
\begin{aligned}
\frac{\partial^{m+n}}{\partial x^{m} \partial y^{n}}\left[\boldsymbol{B}(x) D_{r}\left(y_{0}\right) \boldsymbol{Y}_{r, 2}\left(y_{0}\right)\right] & =(r+1) !\left(\begin{array}{c}
n \\
r+1
\end{array}\right) \sum_{j=0}^{2 r+1-n} \frac{d^{m} B_{j}(x)}{d x^{m}} \times \\
& \times \frac{(r-j) !}{(2 r+1-n-j) !}(-g)^{2 r+1-n-j}
\end{aligned}
$$

for $0 \leq m \leq 2 r+1, r+1 \leq n \leq 2 r+1$;

$$
\begin{aligned}
\frac{\partial^{m+n}}{\partial x^{m} \partial y^{n}}\left[\boldsymbol{B}(x) D_{r}\left(y_{1}\right) \boldsymbol{Y}_{r, 2}\left(y_{1}\right)\right] & =(r+1) ! \sum_{j=0}^{2 r+1-n} \frac{d^{m} B_{j}(x)}{d x^{m}}\left(\begin{array}{c}
n \\
n+j-r
\end{array}\right) \times \\
& \times \frac{(r+1) !}{(2 r+1-n-j)} g^{2 r+1-n-j}
\end{aligned}
$$

for $0 \leq m \leq 2 r+1, r+1 \leq n \leq 2 r+1$. Applying the formulas (2), (3), (4), (5), (6) and (7), (8), (9), (10), the derivatives of $H_{r}(x, y)$ can be calculated and upon these the coefficients $a, b$ can be determined.

Example $2(r=2)$.

$$
\begin{aligned}
H_{2}\left(x_{0}, y_{0}\right) & =\underline{a}_{1}, \\
H_{2}^{(x)}\left(x_{0}, y_{0}\right) & =\underline{a}_{2}, \\
H_{2}^{(x, x)}\left(x_{0}, y_{0}\right) & =\underline{a}_{3} 2, \\
H_{2}^{(y)}\left(x_{0}, y_{0}\right) & =\underline{a}_{4}, \\
H_{2}^{(x, y)}\left(x_{0}, y_{0}\right) & =\underline{a}_{5}, \\
H_{2}^{(y, y)}\left(x_{0}, y_{0}\right) & =\underline{a}_{6} 2, \\
H_{2}\left(x_{1}, y_{0}\right) & =a_{1}+a_{2} h+a_{3} h^{2}+\underline{a}_{7} h^{3}, \\
H_{2}^{(x)}\left(x_{1}, y_{0}\right) & =a_{2}+a_{3} 2 h+a_{7} 3 h^{2}+\underline{a}_{8} h^{3}, \\
H_{2}^{(x, x)}\left(x_{1}, y_{0}\right) & =a_{3} 2+a_{7} 6 h+a_{8} 6 h^{2}+\underline{a}_{9} 2 h^{3}, \\
H_{2}^{(y)}\left(x_{1}, y_{0}\right) & =a_{4}+a_{5} h+\underline{a}_{10} h^{2}, \\
H_{2}^{(x, y)}\left(x_{1}, y_{0}\right) & =a_{5}+a_{10} 2 h+\underline{a}_{11} h^{2}, \\
H_{2}^{(y, y)}\left(x_{1}, y_{0}\right) & =a_{6} 2+\underline{a}_{12} 2 h, \\
H_{2}\left(x_{0}, y_{1}\right) & =a_{1}+a_{4} g+a_{6} g^{2}+\underline{b}_{1} g^{3}, \\
H_{2}^{(x)}\left(x_{0}, y_{1}\right) & =a_{2}+a_{5} g+a_{12} g^{2}+\underline{b}_{2} g^{3}, \\
H_{2}^{(x, x)}\left(x_{0}, y_{1}\right) & =a_{3} 2+a_{10} 2 g-a_{11} 2 h g+\underline{b}_{3} 2 g^{3}, \\
H_{2}^{(y)}\left(x_{0}, y_{1}\right) & =a_{4}+a_{6} 2 g+b_{1} 3 g^{2}+\underline{b}_{4} g^{3},
\end{aligned}
$$




$$
\begin{aligned}
H_{2}^{(x, y)}\left(x_{0}, y_{1}\right)=a_{5} & +a_{12} 2 g+b_{2} 3 g^{2}+\underline{b}_{5} 2 g^{3}, \\
H_{2}^{(y, y)}\left(x_{0}, y_{1}\right)=a_{6} 2 & +b_{1} 6 g+b_{4} 6 g^{2}+\underline{b}_{6} 2 g^{3} \\
H_{2}\left(x_{1}, y_{1}\right)=a_{1} & +a_{2} h+a_{3} h^{2}+a_{4} g+a_{5} h g+a_{6} g^{2}+a_{7} h^{3}+a_{10} h^{2} g+a_{12} h g^{2}+, \\
& +b_{1} g^{3}+b_{2} h g^{3}+b_{3} h^{2} g^{3}+\underline{b}_{7} h^{3} g^{3}, \\
H_{2}^{(x)}\left(x_{1}, y_{1}\right)=a_{2} & +a_{3} 2 h+a_{5} g+a_{7} 3 h^{2}+a_{8} h^{3}+a_{10} 2 h g+a_{11} h^{2} g+a_{12} g^{2}+, \\
& +b_{2} g^{3}+b_{3} 2 h g^{3}+b_{7} 3 h^{2} g^{3}+\underline{b}_{8} h^{3} g^{3} \\
H_{2}^{(x, x)}\left(x_{1}, y_{1}\right)=a_{3} 2 & +a_{7} 6 h+a_{8} 6 h^{2}+a_{9} 2 h^{3}+a_{10} 2 g+a_{11} 4 h g+b_{3} 2 g^{3}+b_{7} 6 h g^{3}+, \\
& +b_{8} 6 h^{2} g^{3}+\underline{b}_{9} 2 h^{3} g^{3} \\
H_{2}^{(y)}\left(x_{1}, y_{1}\right)=a_{4} & +a_{5} h+a_{6} 2 g+a_{10} h^{2}+a_{12} 2 h g+b_{1} 3 g^{2}+b_{2} 3 h g^{2}+b_{3} 3 h^{2} g^{2}+, \\
& +b_{4} g^{3}+b_{5} h g^{3}+b_{7} 3 h^{3} g^{2}+\underline{b}_{10} h^{2} g^{3} \\
H_{2}^{(x, y)}\left(x_{1}, y_{1}\right)=a_{5} & +a_{10} 2 h+a_{11} h^{2}+a_{12} 2 g+b_{2} 3 g^{2}+b_{3} 6 h g^{2}+b_{5} g^{3}+b_{7} 9 h^{2} g^{2}+, \\
& +b_{8} 3 h^{3} g^{2}+b_{10} 2 h g^{3}+\underline{b}_{11} h^{2} g^{3}, \\
H_{2}^{(y, y)}\left(x_{1}, y_{1}\right)=a_{6} 2 & +a_{12} 2 h+b_{1} 6 g+b_{2} 6 h g+b_{3} 6 h^{2} g+b_{4} 6 g^{2}+b_{5} 6 h g^{2}+b_{6} 2 g^{3}+, \\
& +b_{7} 6 h^{3} g+b_{10} 6 h^{2} g^{2}+\underline{b}_{12} 2 h g^{3} .
\end{aligned}
$$

\section{Three-dimensional Polynomial}

The aim of this section is to construct a polynomial $H_{r}(x, y, z)$ which fits the given values on to the coordinate points $x_{0}, x_{1}, y_{0}, y_{1}, z_{0}, z_{1}$ up to its $r$ th derivatives. The conditions can be written as follows:

$$
\frac{\partial^{m+n+p} H_{r}\left(x_{l}, y_{s}, z_{t}\right)}{\partial x^{m} \partial y^{n} \partial z^{p}}=\frac{\partial^{m+n+p} u\left(x_{l}, y_{s}, z_{t}\right)}{\partial x^{m} \partial y^{n} \partial z^{p}}
$$

for $l, s, t=0,1 ; 0 \leq m, n, p \leq r ; 0 \leq m+n+p \leq r$. The number of the $j$ th derivatives of a function having three independent variables is the number of conditions at one point, $s_{j}=\frac{1}{2}\left(j^{2}+3 j\right)+1$; up to its $r$ th derivatives, the number is equal to $S_{r}=$ $\sum_{j=0}^{r} s_{j}=\frac{1}{6}\left(r^{3}+6 r^{2}+11 r+6\right)$, and for the eight points we have $8 S_{r}$.

Let us seek for it in the form

$$
H_{r}(x, y, z)=\boldsymbol{A}(x, z) \boldsymbol{Y}_{r, 1}(y)+\boldsymbol{B}(x, z) D_{r}(y) \boldsymbol{Y}_{r, 2}(y) .
$$

For the sake of a convenient form of writing the vectors, let us define the unit vector of $j+1$ entries $\boldsymbol{e}_{j, q+1}(0 \leq q \leq j)$ the $q+1$-th entry of which is 1 , and all the 
rest are zero. Then the vectors in $H_{r}(x, y, z)$ can be written by using the formulas

$$
\begin{aligned}
& \boldsymbol{Y}_{j, 1}(y)=\sum_{i=0}^{j}\left(y-y_{0}\right)^{i} \boldsymbol{e}_{j, i+1}, \\
& \boldsymbol{Y}_{j, 2}(y)=\sum_{i=0}^{j}\left(y-y_{1}\right)^{i} \boldsymbol{e}_{j, i+1}, \\
& D_{j}(y)=\left(y-y_{0}\right)^{j+1}, \quad 0 \leq j \leq r . \\
& \boldsymbol{A}(x, z)=\sum_{j=0}^{r} A_{j}(x, z) \boldsymbol{e}_{r, r+1-j}, \\
& \boldsymbol{B}(x, z)=\sum_{j=0}^{r} B_{j}(x, z) \boldsymbol{e}_{r, r+1-j}, \\
& A_{j}(x, z)=\boldsymbol{A}_{j, 1}(z) \boldsymbol{X}_{j, 1}(x)+\boldsymbol{A}_{j, 2}(z) C_{j}(x) \boldsymbol{X}_{j, 2}(x), \quad 0 \leq j \leq r, \\
& B_{j}(x, z)=\boldsymbol{B}_{j, 1}(z) \boldsymbol{X}_{j, 1}(x)+\boldsymbol{B}_{j, 2}(z) C_{j}(x) \boldsymbol{X}_{j, 2}(x), \quad 0 \leq j \leq r, \\
& \boldsymbol{X}_{j, 1}(x)=\sum_{i=0}^{j}\left(x-x_{0}\right)^{i} \boldsymbol{e}_{j, i+1}, \\
& \boldsymbol{X}_{j, 2}(x)=\sum_{i=0}^{j}\left(x-x_{1}\right)^{i} \boldsymbol{e}_{j, i+1}, \\
& C_{j}(x)=\left(x-x_{0}\right)^{j+1}, \quad 0 \leq j \leq r, \\
& \boldsymbol{A}_{j, 1}(z)=\sum_{q=0}^{j} A_{j, 1, q}(z) \boldsymbol{e}_{j, j+1-q}, \\
& \boldsymbol{A}_{j, 2}(z)=\sum_{q=0}^{j} A_{j, 2, q}(z) \boldsymbol{e}_{j, j+1-q}, \\
& \boldsymbol{B}_{j, 1}(z)=\sum_{q=0}^{j} B_{j, 1, q}(z) \boldsymbol{e}_{j, j+1-q}, \\
& \boldsymbol{B}_{j, 2}(z)=\sum_{q=0}^{j} B_{j, 2, q}(z) \boldsymbol{e}_{j, j+1-q}, \\
& A_{j, 1, q}(z)=\boldsymbol{\alpha}_{j, 1,2 k} \boldsymbol{Z}_{q, 1}(z)+\boldsymbol{\alpha}_{j, 1,2 k+1} E_{j}(z) \boldsymbol{Z}_{q, 2}(z), \quad 0 \leq q \leq j, \\
& A_{j, 2, q}(z)=\boldsymbol{\alpha}_{j, 2,2 k} \boldsymbol{Z}_{q, 1}(z)+\boldsymbol{\alpha}_{j, 2,2 k+1} E_{j}(z) \boldsymbol{Z}_{q, 2}(z), \quad 0 \leq q \leq j, \\
& B_{j, 1, q}(z)=\boldsymbol{\beta}_{j, 1,2 k} \boldsymbol{Z}_{q, 1}(z)+\boldsymbol{\beta}_{j, 1,2 k+1} E_{j}(z) \boldsymbol{Z}_{q, 2}(z), \quad 0 \leq q \leq j, \\
& B_{j, 2, q}(z)=\boldsymbol{\beta}_{j, 2,2 k} \boldsymbol{Z}_{q, 1}(z)+\boldsymbol{\beta}_{j, 2,2 k+1} E_{j}(z) \boldsymbol{Z}_{q, 2}(z), \quad 0 \leq q \leq j, \\
& l_{r, q}=(r-q) \frac{r+3+q}{2}, \\
& l_{j, q}=(j-q) \frac{r+3+q}{2}+\sum_{i=j+1}^{r} n_{i}, \\
& n_{i}=\frac{1}{2}(i+1)(i+2), \\
& N_{r}=\sum_{i=0}^{r} n_{i}=\frac{1}{6}\left(r^{3}+6 r^{2}+11 r+6\right) \\
& L_{r, q}=2 N_{r}+l_{r, q} \text {, } \\
& L_{j, q}=2 N_{r}+l_{j, q}, \quad k=j-q \text {; } \\
& \boldsymbol{\alpha}_{j, 1,2 k}=\sum_{i=0}^{q} a_{l j, q+i+1} \boldsymbol{e}_{q, i+1} \text {, } \\
& \boldsymbol{\alpha}_{j, 1,2 k+1}=\sum_{i=0}^{q} a_{N_{r}+l_{j, q}+i+1} \boldsymbol{e}_{q, i+1} \text {, }
\end{aligned}
$$




$$
\boldsymbol{\alpha}_{j, 2,2 k}=\sum_{i=0}^{q} a_{L_{j, q}+i+1} \boldsymbol{e}_{q, i+1}, \quad \boldsymbol{\alpha}_{j, 2,2 k+1}=\sum_{i=0}^{q} a_{N_{r}+L_{j, q}+i+1} \boldsymbol{e}_{q, i+1} .
$$

The relations between $\beta$ and $b$ are analogous to the previously given formulas where $a$ is replaced by $b$.

The number of the constants $a, b$ in $\boldsymbol{A}_{j, 1}, \boldsymbol{A}_{j, 2}, \boldsymbol{B}_{j, 1}, \boldsymbol{B}_{j, 2}$ is $8 N_{r}$, which agrees with the number of conditions $8 N_{r}=8 S_{r}$. Consequently, $H_{r}(x, y, z)$ is the minimal degree polynomial satisfying the requirements.

The distances between the coordinates are $x_{1}-x_{0}=h, y_{1}-y_{0}=g, z_{1}-z_{0}=l$. The derivatives of the $H_{r}(x, y, z)$ function are needed to compute the coefficients $a$ and $b$ (rather tedious calculations are omitted here to save space):

$$
\begin{aligned}
& \frac{d^{p} A_{j, 1, q}\left(z_{0}\right)}{d z^{p}}= p ! a_{l_{j, q}+p+1}, \quad 0 \leq p \leq q, \\
& \frac{d^{p} A_{j, 1, q}\left(z_{1}\right)}{d z^{p}}= \sum_{i=0}^{q-p} \frac{(p+i) !}{i !} l^{i} a_{l_{j, q}+p+i+1}+ \\
& \quad+p !(q+1) ! \sum_{i=0}^{p} \frac{l^{q+1-p+i} a_{N_{r}+l_{j, q}+i+1}}{(p-i) !(q+1-p+i) !}, \quad 0 \leq p \leq q, \\
& \frac{d^{p} A_{j, 1, q}\left(z_{0}\right)}{d z^{p}}=\left(\begin{array}{c}
p-(q+1) \\
p
\end{array}\right)(q+1) ! \sum_{i=p-(q+1)}^{q} \frac{i !(-l)^{q+1-p+i}}{(q+1-p+i) !} a_{N_{r}+l_{j, q}+i+1}, \\
& \frac{d^{p} A_{j, 1, q}\left(z_{1}\right)}{d z^{p}}= p !(q+1) ! \sum_{i=p-(q+1)}^{q} \frac{l^{q+1-p+i}}{(p-i) !(q+1-p+i) !} a_{N_{r}+l_{j, q}+i+1}, \\
& q+1 \leq p \leq 2 q+1 .
\end{aligned}
$$

The derivatives of (11) are similar to the previous ones,

$$
\begin{aligned}
\frac{d^{p} A_{j, 2, q}\left(z_{0}\right)}{d z^{p}}= & p ! a_{L_{j, q}+p+1} \\
\frac{d^{p} A_{j, 2, q}\left(z_{1}\right)}{d z^{p}}= & \sum_{i=0}^{q-p} \frac{(p+i) !}{i !} l^{i} a_{L_{j, q}+p+i+1}+ \\
& \quad+p !(q+1) ! \sum_{i=0}^{p} \frac{l^{q+1-p+i} a_{N_{r}+L_{j, q}+i+1}}{(p-i) !(q+1-p+i) !}, \quad 0 \leq p \leq q \\
\frac{d^{p} A_{j, 2, q}\left(z_{0}\right)}{d z^{p}}= & \quad \underset{p-(q+1)}{p})(q+1) ! \sum_{i=p-(q+1)}^{q} \frac{i !(-l)^{q+1-p+i} a_{N_{r}+L_{j, q}+i+1}}{(q+1-p+i) !} \\
\frac{d^{p} A_{j, 2, q}\left(z_{1}\right)}{d z^{p}}= & p !(q+1) ! \sum_{i=p-(q+1)}^{q} \frac{l^{q+1-p+i} a_{N_{r}+L_{j, q}+i+1}}{(p-i) !(q+1-p+i) !}, \quad q+1 \leq p \leq 2 q+1
\end{aligned}
$$


To obtain similar formulas for $B_{j, 1, q}$ and $B_{j, 2, q}, a$ should be replaced by $b$.

For the derivatives of $A_{j}(x, z)$, expressions (11), (12), (13), (14) are to be used,

$$
\begin{aligned}
& \frac{\partial^{m+p}}{\partial x^{m} \partial z^{p}}\left[\boldsymbol{A}_{j, 1}(z) \boldsymbol{X}_{j, 1}\left(x_{0}\right)\right]=0 \quad \text { for } m \neq j-q, \\
& \frac{\partial^{m+p}}{\partial x^{m} \partial z^{p}}\left[\boldsymbol{A}_{j, 1}(z) \boldsymbol{X}_{j, 1}\left(x_{0}\right)\right]=\frac{d^{p} A_{j, 1, j-m}(z)}{d z^{p}} m ! \\
& \frac{\partial^{m+p}}{\partial x^{m} \partial z^{p}}\left[\boldsymbol{A}_{j, 1}(z) \boldsymbol{X}_{j, 1}\left(x_{1}\right)\right]=\sum_{q=0}^{j-m} \frac{d^{p} A_{j, 1, q}(z)}{d z^{p}} \frac{(j-q) ! h^{j-q-m}}{(j-q-m) !}, \quad 0 \leq m \leq j ; \\
& \frac{\partial^{m+p}}{\partial x^{m} \partial z^{p}}\left[\boldsymbol{A}_{j, 2}(z) C_{j}\left(x_{0}\right) \boldsymbol{X}_{j, 2}\left(x_{0}\right)\right]=0, \quad 0 \leq m \leq j, \\
& \frac{\partial^{m+p}}{\partial x^{m} \partial z^{p}}\left[\boldsymbol{A}_{j, 2}(z) C_{j}\left(x_{1}\right) \boldsymbol{X}_{j, 2}\left(x_{1}\right)\right]=\sum_{q=j-m}^{j} \frac{d^{p} A_{j, 2, q}(z)}{d z^{p}} \times \\
& \times \frac{m !(j+1) ! h^{2 j+1-m-q}}{(m-(j-q)) !(2 j+1-m-q) !}, \quad 0 \leq m \leq j, j-q<m . \\
& \frac{\partial^{m+p}}{\partial x^{m} \partial z^{p}}\left[\boldsymbol{A}_{j, 2}(z) C_{j}\left(x_{0}\right) \boldsymbol{X}_{j, 2}\left(x_{0}\right)\right]=\frac{(m) !}{(m-(j+1)) !} \times \\
& \times \sum_{q=0}^{2 j+1-m} \frac{d^{p} A_{j, 2, q}(z)}{d z^{p}} \frac{(j-q) !(-h)^{2 j+1-m-q}}{(2 j+1-m-q) !}, \\
& \frac{\partial^{m+p}}{\partial x^{m} \partial z^{p}}\left[\boldsymbol{A}_{j, 2}(z) C_{j}\left(x_{1}\right) \boldsymbol{X}_{j, 2}\left(x_{1}\right)\right]=m !(j+1) ! \times \\
& \times \sum_{q=0}^{2 j+1-m} \frac{d^{p} A_{j, 2, q}(z)}{d z^{p}} \frac{h^{2 j+1-m-q}}{(m-(j-q)) !(2 j+1-m-q)},
\end{aligned}
$$

where $j+1 \leq m \leq 2 j+1$. The derivatives of $B_{j}(x, z)$ will be obtained by replacing $A$ by $B$ in formulas (15), (16), and (17).

Finally, the derivatives of the first and second terms in the Hermite polynomials are to be given, and these are as follows:

$$
\begin{aligned}
\frac{\partial^{m+n+p}}{\partial x^{m} \partial y^{n} \partial z^{p}}\left[\boldsymbol{A}(x, z) \boldsymbol{Y}_{r, 1}\left(y_{0}\right)\right] & =n ! \frac{\partial^{m+p} A_{r-n}(x, z)}{\partial x^{m} \partial z^{p}}, \\
\frac{\partial^{m+n+p}}{\partial x^{m} \partial y^{n} \partial z^{p}}\left[\boldsymbol{A}(x, z) \boldsymbol{Y}_{r, 1}\left(y_{1}\right)\right] & =\sum_{j=0}^{r-n} \frac{\partial^{m+p} A_{j}(x, z)}{\partial x^{m} \partial z^{p}} \frac{(r-j) ! g^{r-j-n}}{(r-j-n) !}, \quad 0 \leq n \leq r ; \\
\frac{\partial^{m+n+p}}{\partial x^{m} \partial y^{n} \partial z^{p}}\left[\boldsymbol{B}(x, z) D_{r}\left(y_{0}\right) \boldsymbol{Y}_{r, 2}\left(y_{0}\right)\right] & =0, \quad 0 \leq n \leq r,
\end{aligned}
$$




$$
\begin{gathered}
\frac{\partial^{m+n+p}}{\partial x^{m} \partial y^{n} \partial z^{p}}\left[\boldsymbol{B}(x, z) D_{r}\left(y_{1}\right) \boldsymbol{Y}_{r, 2}\left(y_{1}\right)\right]=(r+1) ! \sum_{j=r-n}^{r} \frac{\partial^{m+p} B_{j}(x, z)}{\partial x^{m} \partial z^{p}} \times \\
\times\left(\begin{array}{c}
n \\
n+j-r
\end{array}\right) \frac{(r-j) ! g^{2 r+1-n-j}}{(2 r+1-n-j) !}, \quad 0 \leq n \leq r ; \\
\begin{array}{c}
\frac{\partial^{m+n+p}}{\partial x^{m} \partial y^{n} \partial z^{p}}\left[\boldsymbol{B}(x, z) D_{r}\left(y_{0}\right) \boldsymbol{Y}_{r, 2}\left(y_{0}\right)\right]=\left(\begin{array}{c}
n \\
r+1
\end{array}\right)(r+1) ! \sum_{j=0}^{2 r+1-n} \frac{\partial^{m+p} B_{j}(x, z)}{\partial x^{m} \partial z^{p}} \times \\
(2 r+1-n-j) !(-g)^{2 r+1-n-j}
\end{array} \\
\frac{\partial^{m+n+p}}{\partial x^{m} \partial y^{n} \partial z^{p}}\left[\boldsymbol{B}(x, z) D_{r}\left(y_{1}\right) \boldsymbol{Y}_{r, 2}\left(y_{1}\right)\right]=(r+1) ! \sum_{j=0}^{2 r+1-n} \frac{\partial^{m+p} B_{j}(x, z)}{\partial x^{m} \partial z^{p}}\left(\begin{array}{c}
n \\
n+j-r
\end{array}\right) \times \\
\times \frac{(r-j) ! g^{2 r+1-n-j}}{(2 r+1-n-j) !}, \quad r+1 \leq n \leq 2 r+1 .
\end{gathered}
$$

Example $3(r=2)$. In order to save space, we show below only the first 40 coefficients:

$$
\begin{aligned}
H_{2}\left(x_{0}, y_{0}, z_{0}\right) & =\underline{a}_{1}, \\
H_{2}^{(z)}\left(x_{0}, y_{0}, z_{0}\right) & =\underline{a}_{2}, \\
H_{2}^{(z, z)}\left(x_{0}, y_{0}, z_{0}\right) & =\underline{a}_{3} 2 \\
H_{2}^{(x)}\left(x_{0}, y_{0}, z_{0}\right) & =\underline{a}_{4}, \\
H_{2}^{(x, z)}\left(x_{0}, y_{0}, z_{0}\right) & =\underline{a}_{5}, \\
H_{2}^{(x, x)}\left(x_{0}, y_{0}, z_{0}\right) & =\underline{a}_{6} 2 \\
H_{2}^{(y)}\left(x_{0}, y_{0}, z_{0}\right) & =\underline{a}_{7}, \\
H_{2}^{(y, z)}\left(x_{0}, y_{0}, z_{0}\right) & =\underline{a}_{8}, \\
H_{2}^{(x, y)}\left(x_{0}, y_{0}, z_{0}\right) & =\underline{a}_{9}, \\
H_{2}^{(y, y)}\left(x_{0}, y_{0}, z_{0}\right) & =\underline{a}_{10} 2, \\
H_{2}\left(x_{0}, y_{0}, z_{1}\right) & =a_{1}+a_{2} l+a_{3} l^{2}+\underline{a}_{11} l^{3}, \\
H_{2}^{(z)}\left(x_{0}, y_{0}, z_{1}\right) & =a_{2}+a_{3} 2 l+a_{11} 3 l^{2}+\underline{a}_{12} l^{3}, \\
H_{2}^{(z, z)}\left(x_{0}, y_{0}, z_{1}\right) & =a_{3} 2+a_{11} 6 l+a_{12} 6 l^{2}+\underline{a}_{13} 2 l^{3}, \\
H_{2}^{(x)}\left(x_{0}, y_{0}, z_{1}\right) & =a_{4}+a_{5} l+\underline{a}_{14} l^{2}, \\
H_{2}^{(x, z)}\left(x_{0}, y_{0}, z_{1}\right) & =a_{5}+a_{14} 2 l+\underline{a}_{15} l^{2}, \\
H_{2}^{(x, x)}\left(x_{0}, y_{0}, z_{1}\right) & =a_{6} 2+\underline{a}_{16} 2 l,
\end{aligned}
$$




$$
\begin{aligned}
& H_{2}^{(y)}\left(x_{0}, y_{0}, z_{1}\right)=a_{7}+a_{8} l+\underline{a}_{17} l^{2}, \\
& H_{2}^{(y, z)}\left(x_{0}, y_{0}, z_{1}\right)=a_{8}+a_{17} 2 l+\underline{a}_{18} l^{2}, \\
& H_{2}^{(x, y)}\left(x_{0}, y_{0}, z_{1}\right)=a_{9}+\underline{a}_{19} l, \\
& H_{2}^{(y, y)}\left(x_{0}, y_{0}, z_{1}\right)=a_{10} 2+\underline{a}_{20} 2 l, \\
& H_{2}\left(x_{1}, y_{0}, z_{0}\right)=a_{6} h^{2}+a_{4} h+a_{1}+\underline{a}_{21} h^{3}, \\
& H_{2}^{(z)}\left(x_{1}, y_{0}, z_{0}\right)=a_{16} h^{2}+a_{5} h+a_{2}+\underline{a}_{22} h^{3} \text {, } \\
& H_{2}^{(z, z)}\left(x_{1}, y_{0}, z_{0}\right)=\left(a_{14} 2-a_{15} 2 l\right) h+a_{3} 2+\underline{a}_{23} 2 h^{3} \text {, } \\
& H_{2}^{(x)}\left(x_{1}, y_{0}, z_{0}\right)=a_{6} 2 h+a_{4}+a_{21} 3 h^{2}+\underline{a}_{24} h^{3} \text {, } \\
& H_{2}^{(x, z)}\left(x_{1}, y_{0}, z_{0}\right)=a_{16} 2 h+a_{5}+a_{22} 3 h^{2}+\underline{a}_{25} h^{3} \text {, } \\
& H_{2}^{(x, x)}\left(x_{1}, y_{0}, z_{0}\right)=a_{6} 2+a_{21} 6 h+a_{24} 6 h^{2}+\underline{a}_{26} h^{3} \text {, } \\
& H_{2}^{(y)}\left(x_{1}, y_{0}, z_{0}\right)=a_{9} h+a_{7}+\underline{a}_{27} h^{2}, \\
& H_{2}^{(y, z)}\left(x_{1}, y_{0}, z_{0}\right)=a_{19} h+a_{8}+\underline{a}_{28} h^{2}, \\
& H_{2}^{(x, y)}\left(x_{1}, y_{0}, z_{0}\right)=a_{9}+a_{27} 2 h+\underline{a}_{29} h^{2}, \\
& H_{2}^{(y, y)}\left(x_{1}, y_{0}, z_{01}\right)=\left(a_{10}+\underline{a}_{30} h\right) 2 \text {, } \\
& H_{2}\left(x_{1}, y_{0}, z_{1}\right)=a_{1}+a_{2} l+a_{3} l^{2}+a_{11} l^{3}+\left(a_{4}+a_{5} l+a_{14} l^{2}\right) h+\left(a_{6}+a_{16} l\right) h^{2}+, \\
& +\left(a_{21}+a_{22} l+a_{23} l^{2}+\underline{a}_{31} l^{3}\right) h^{3}, \\
& H_{2}^{(z)}\left(x_{1}, y_{0}, z_{1}\right)=a_{2}+a_{3} 2 l+a_{11} 3 l^{2}+a_{12} l^{3}+a_{16} h^{2}+\left(a_{5}+a_{14} 2 l+a_{15} l^{2}\right) h+\text {, } \\
& +\left(a_{22}+a_{23} 2 l+a_{31} 3 l^{2}+\underline{a}_{32} l^{3}\right) h^{3}, \\
& H_{2}^{(z, z)}\left(x_{1}, y_{0}, z_{1}\right)=a_{3} 2+a_{11} 6 l+a_{12} 6 l^{2}+a_{13} 2 l^{3}+\left(a_{14} 2+a_{15} 4 l\right) h+, \\
& +\left(a_{23} 2+a_{31} 6 l+a_{32} 6 l^{2}+\underline{a}_{33} 2 l^{3}\right) h^{3}, \\
& H_{2}^{(x)}\left(x_{1}, y_{0}, z_{1}\right)=a_{4}+a_{5} l+a_{14} l^{2}+\left(a_{6}+a_{16} l\right) 2 h+, \\
& \left(a_{21}+a_{22} l+a_{23} l^{2}+a_{31} l^{3}\right) 3 h^{2}+\left(a_{24}+a_{25} l+\underline{a}_{34} l^{2}\right) h^{3}, \\
& H_{2}^{(x, z)}\left(x_{1}, y_{0}, z_{1}\right)=a_{5}+a_{14} 2 l+a_{15} l^{2}+a_{16} 2 h+, \\
& +\left(a_{22}+a_{23} 2 l+a_{31} 3 l^{2}+a_{32} l^{3}\right) 3 h^{2}+\left(a_{25}+a_{34} 2 l+\underline{a}_{35} l^{2}\right) h^{3}, \\
& H_{2}^{(x, x)}\left(x_{1}, y_{0}, z_{1}\right)=a_{6} 2+a_{16} 2 l+\left(a_{24}+a_{25} l+a_{34} l^{2}\right) 6 h^{2}+, \\
& +\left(a_{21}+a_{22} l+a_{23} l^{2}+a_{31} l^{3}\right) 6 h+\left(a_{26}+\underline{a}_{36} l\right) h^{3}, \\
& H_{2}^{(y)}\left(x_{1}, y_{0}, z_{1}\right)=a_{7}+a_{8} l+a_{17} l^{2}+\left(a_{9}+a_{19} l\right) h+,
\end{aligned}
$$




$$
\begin{aligned}
&+\left(a_{27}+a_{28} l+\underline{a}_{37} l^{2}\right) h^{2}, \\
& H_{2}^{(y, z)}\left(x_{1}, y_{0}, z_{1}\right)=a_{8}+a_{17} 2 l+a_{18} l^{2}+a_{19} h+, \\
&+\left(a_{28}+a_{37} 2 l+\underline{a}_{38} l^{2}\right) h^{2}, \\
& H_{2}^{(x, y)}\left(x_{1}, y_{0}, z_{1}\right)=a_{9}+a_{19} l+\left(a_{27}+a_{28} l+a_{37} l^{2}\right) 2 h+\left(a_{29}+\underline{a}_{39} l\right) h^{2}, \\
& H_{2}^{(y, y)}\left(x_{1}, y_{0}, z_{1}\right)=a_{10} 2+a_{20} 2 l+\left(a_{30}+\underline{a}_{40} l\right) 2 h .
\end{aligned}
$$

\section{REFERENCES}

[1] Gel'Fond, A. O.: Calculus of Finite Differences, Hindustan Publ. Co., Dehli, 1971 (English translation).

[2] Jordan, Сн.: Calculus of Finite Differences, Hungarian Agent Eggenberger Book-Shop, Budapest, 1939.

[3] LoRentz, R. A.: Multivariate Hermite interpolation by algebraic polynomials: A survey, J. Comput. Appl. Math., 122 (2000), No. 1-2, 67-201.

[4] Natanson, L. P.: Constructive Function Theory, Vol. III, Frederic Ungar Publ. Co., New York, 1965 (English translation).

[5] VARgA, R. S.: Functional Analysis and Approximation Theory in Numerical Analysis, CBMS-NSF Regional Conference Series in Applied Mathematics, SIAM, Philadelphia, 1971.

[6] Whittaker, E. and Robinson, G.: The Calculus of Observations. A Treatise on Numerical Mathematics, 4th ed., Blackie \& Son Ltd., London \& Glasgow, 1954.

\section{Author's address}

\section{A. Nyíri:}

University of Miskolc, H-3515 Miskolc-Egyetemváros, Hungary

E-mail address: aramnya@uni-miskolc.hu 